\title{
Melanin Through Deep Time: Experimental and Analytical Approaches to Decoding the Fossil Record of Melanin
}

\author{
Maria McNamara \\ University College Cork, Ireland, Herndon, Virginia, United States \\ Spectacular discoveries of evidence of melanin pigments in fossils over the last 12 years have driven the \\ birth of a new field in palaeontology, i.e. that of fossil colour. But how do we know that we can trust the \\ fossil evidence? In this seminar I will review current developments in this emerging field, highlighting \\ key controversies and developments. I will illustrate how a multifaceted approach incorporating \\ electrobeam analysis, synchrotron analysis, mass spectrometry and vibrational spectroscopy is critical to \\ the identification of fossil melanin. Ultimately, our ability to interpret evidence of colour in fossils is \\ controlled by our understanding of fossil preservation.
}

